\title{
On the fixed-time stabilization of input delay systems using act-and-wait control
}

\author{
Wim Michiels ${ }^{\mathrm{a}, *}$, Bin Zhou ${ }^{\mathrm{b}}$ \\ ${ }^{a}$ Department of Computer Science, KU Leuven, B-3001 Heverlee, Belgium \\ ${ }^{b}$ Center for Control Theory and Guidance Technology, Harbin Institute of Technology, Harbin, China
}

\begin{abstract}
We address the state feedback stabilization of linear systems with input delay using the so-called actand-wait control strategy. The latter approach induces an analysis of the closed-loop stability based on a finite-dimensional monodromy operator, and rendering this matrix nilpotent results in fixed-time stability. We show that any controllable planar system can be stabilized in a fixed time, and the constructive proof reveals that always two isolated solutions for the corresponding controller gain co-exist. Next, for the fixed-time stabilization of general linear systems, we present both a novel numerical approach for the computation of the controller gain, and an analytic approach relying on incorporating a predictor and an appropriately defined feedback transformation in the control scheme. Several illustrations complete the presentation.
\end{abstract}

Keywords: finite-time stabilization, time-delay systems, act-and-wait control

\section{Introduction}

We consider the fixed-time stabilization of the linear system

$$
\dot{x}(t)=A x(t)+B u(t-\tau)
$$

where $x(t) \in \mathbb{R}^{n}$ is the state-variable at time $t$, $u(t) \in \mathbb{R}^{m}$ is the input at time $t$, and $\tau$ is an input delay, using a feedback controller. Throughout the paper we make the following assumption.

Assumption 1. The pair $(A, B)$ is controllable and $m<n$.

The design of stabilizing controllers having a finite convergence time, known as finite-time stabilization, has received increased interest in the last two decades. Unlike traditional stabilization methods, by which the closed-loop system converges to zero asymptotically, the finite-time control drives the closed-loop system state to rest in a finite time. Several effective methods have been developed for achieving finite-time stability, including methods based on finite-time linear quadratic regulation with fixed terminal constraints [10], on sliding mode control [17], on Lyapunov differential inequalities $[2,22]$, on the concept of homogeneity

\footnotetext{
*Corresponding author

Email addresses: Wim.Michiels@cs. kuleuven.be (Wim Michiels), binzhoulee@163.com, binzhou@hit .edu.cn (Bin Zhou)
}

$[3,5,6]$ (see also $[18,19]$ for its use to design distributed finite-time observers), and based on an implicit Lyapunov function [15]. For these methods the convergence time generally depends on the initial condition. For some classes of systems, it is also possible to achieve convergence to zero in a time independent of initial conditions, referred to as fixed-time stability. Such a problem was solved in [14], based on Lyapunov differential inequalities. Further advances were made in [20] using time-varying feedback for nonlinear systems in the normal form, where the convergence time can be prescribed in advance. Fixed-time stabilizing designs were also sought after in $[25,26]$ for general linear systems, by solving parametric Lyapunov equations or nonlinear differential equations, resulting in bounded linear time-varying high-gain feedback.

As a distinctive feature with respect to the above works, we employ a linear periodic feedback controller of the form

$$
u(t)=-g_{\tau}(t) K x(t),
$$

with $g_{\tau}: \mathbb{R} \rightarrow \mathbb{R}$ a function with period $2 \tau$ and

$$
g_{\tau}(t)= \begin{cases}0, & t \in[0, \tau], \\ 1, & t \in(\tau, 2 \tau),\end{cases}
$$

which corresponds to a realization of the act-andwait control strategy. This strategy was introduced by Insperger and Stépán in [7, 21] (albeit for systems with delay in the measured output), and later 
on it was extended to the time-delayed control of nonlinear systems $[8,16]$. The closed-loop system (1)-(2) is a time-periodic time-delay systems. An established approach to assess the stability for linear periodic delay equations consists of computing dominant eigenvalues of the monodromy operator, which is in general an infinite-dimensional operator. However, the shape of function (3) implies that the control is switched on and off (i.e., there is an alternation between an act and a wait phase) and due to the open loop phases of duration $\tau$, stability can still be assessed from the spectrum of an $n \times n$ monodromy matrix. The latter directly offers potential of direct pole placement, yet a challenge lies in the nonlinear dependence of the characteristic function on the gain parameters.

In order to give a more precise problem statement, we consider the initial value problem for (1)-(2) at time $t=0$, by adding initial conditions $\left(x_{0}, u_{0}\right) \in X:=\mathbb{R}^{n} \times \mathcal{P C}\left([-\tau, 0], \mathbb{R}^{m}\right)$, where $\mathcal{P} C\left([-\tau, 0], \mathbb{R}^{m}\right)$ is the space of piecewise continuous function from $[-\tau, 0]$ to $\mathbb{R}^{m}$. For every $\left(x_{0}, u_{0}\right) \in X$ the emanating solution is uniquely defined, which we denote by $x\left(\cdot ; x_{0}, u_{0}\right)$ and $u\left(\cdot ; x_{0}, u_{0}\right)$. The closed-loop system (1)-(2) is said to be fixed-time stable with convergence time $T$ if it is stable and

$$
x\left(t ; x_{0}, u_{0}\right)=0, \forall t \geq T, \forall\left(x_{0}, u_{0}\right) \in X .
$$

Throughout the paper we address the following problem.

Problem 1. Design a feedback gain $K$ such that the closed-loop system consisting of (1) and (2) is fixed-time stable, and characterize the convergence time $T$.

The motivation for studying Problem 1, on the one hand, comes from the case studies in [7, 24] showing that achieving fixed-time stability is possible, and, on the other hand, from the article [27], where it is shown that using a smooth periodically varying gain it is possible to render the monodromy matrix equal to the zero matrix. Note that using control law (2), finite-time stability is imposed by a time-dependent switching rather than a state-dependent switching as in a sliding modes solution.

The structure of the paper is as follows. In Section 2 we explain the fixed-time stabilization mechanism using act-and-wait control, and we shed a light on the limitations and the potential of control law (2). In Section 3 we provide a constructive proof for the fixed-time stabilizability of planar systems. In Section 4 we present two general design methodologies: a numerical approach allowing to compute the gains $K$ in (2) for solving Problem 1 whenever they exist, as well as an analytic approach guaranteeing fixed-time convergence, which is based on augmenting the closedloop system with a predictor and a preliminary feedback transformation. In Section 5 we illustrate the obtained results, and in Section 6 we formulate some concluding comments.

\section{Motivation and preliminaries}

Control law (3) is a special instance of the periodic feedback

$$
u(t)=-L(t) x(t),
$$

where $L: \mathbb{R} \rightarrow \mathbb{R}^{m \times n}$ is a $2 \tau$-periodic function satisfying $L(t)=0$ for $t \in[0, \tau]$. To understand the stabilization mechanism, we first note that, due to the controllability of $(A, B)$, the value of $x(\tau)$ is freely assignable by an appropriate choice of initial condition $\left(x_{0}, u_{0}\right) \in X$. For $t \geq \tau$ the closed-loop dynamics are determined by

$$
\dot{x}(t)=A x(t)-B L(t-\tau) x(t-\tau) .
$$

In the interval $[\tau, 2 \tau]$, we have $L(t-\tau)=0$ and system (1) works in open loop mode, hence, we can express

$$
x(t)=\mathrm{e}^{A(t-\tau)} x(\tau), t \in[\tau, 2 \tau] .
$$

In the interval $[2 \tau, 3 \tau]$ the dynamics are described by

$$
\begin{aligned}
\dot{x}(t) & =A x(t)-B L(t-\tau) x(t-\tau) \\
& =A x(t)-B L(t-\tau) \mathrm{e}^{A(t-2 \tau)} x(\tau)
\end{aligned}
$$

and using the variable of constants formula we arrive at

$$
x(3 \tau)=\mathcal{M} x(\tau),
$$

with $\mathcal{M}$ the monodromy matrix,

$$
\mathcal{M}:=\mathrm{e}^{A \tau}\left[\mathrm{e}^{A \tau}-\int_{0}^{\tau} \mathrm{e}^{-A s} B L(s+\tau) \mathrm{e}^{A s} \mathrm{~d} s\right] .
$$

By the periodicity of the controller gain we get

$$
x((2 k+1) \tau)=\mathcal{M} x((2 k-1) \tau), k \geq 1,
$$

while $x((2 k+1) \tau)=0$ for some $k \in \mathbb{N}$ implies that $x(t)=0$ for all $t \geq(2 k+1) \tau$. Hence, we arrive at the following result.

Proposition 1. System (1) and (4) is fixed-time stable if and only if matrix $\mathcal{M}$ is nilpotent. Furthermore, if $\mathcal{M}$ is nilpotent with index $v \in\{1, \ldots, n\}$, i.e.

$$
\mathcal{M}^{\nu-1} \neq 0, \quad \mathcal{M}^{v}=0,
$$

then the convergence time is given by $T=(1+2 v) \tau$. 
In [27, Theorem 1] it was shown that the periodic gain $L(t)$ can always be chosen such that fixed-time stability with convergence time $T=3 \tau$ can be achieved. More precisely, choosing $R_{\tau}$ : $\mathbb{R} \rightarrow \mathbb{R}^{m \times m}$ to be a continuous $2 \tau$-periodic function satisfying $R_{\tau}(t) \geq 0$ for all $t \in[0,2 \tau], R_{\tau}(t)=0$ for $t \in[0, \tau]$ and $R_{\tau}\left(t^{*}\right)>0$ for some $t^{*} \in(\tau, 2 \tau)$, and letting

$$
W:=\int_{0}^{\tau} \mathrm{e}^{A(\tau-s)} B R_{\tau}(s+\tau) B^{\mathrm{T}} \mathrm{e}^{-A^{\mathrm{T}}(\tau-s)} \mathrm{d} s>0,
$$

the control law

$$
u(t)=-\underbrace{R_{\tau}(t) B^{\mathrm{T}} \mathrm{e}^{A^{\mathrm{T}}(2 \tau-t)} W^{-1} \mathrm{e}^{A(2 \tau-t)}}_{L(t)} x(t),
$$

results in $\mathcal{M}=0$, which can be verified by substitution into (5). As another distinctive property, a desired level of smoothness on the gain $L(t)$ can be imposed by the choice of $R_{\tau}$ [27].

Because the control law (2), which only depends on $n$ discrete gain parameters and results in monodromy matrix

$$
\Delta(K):=\mathrm{e}^{A \tau}\left[\mathrm{e}^{A \tau}-\int_{0}^{\tau} \mathrm{e}^{-A s} B K \mathrm{e}^{A s} \mathrm{~d} s\right],
$$

is more structurally constrained than (4), it is in general not possible to impose a zero monodromy matrix by an appropriate choice of $K$, inducing a solution to Problem 1 with $T=3 \tau$. This is clarified in the following proposition.

Proposition 2. Assume that all eigenvalues of $A$ are simple and denote them by $\lambda_{i}, i=1,2, \ldots, n$. The following assertions hold.

\section{If condition}

$$
\mathrm{e}^{\left(\lambda_{i}-\lambda_{j}\right) \tau} \neq 1, \quad \forall i, j \in\{1, \ldots, n\}
$$

is satisfied, then Problem 1 with $T=3 \tau$ is not solvable using (3);

\section{If condition}

$$
\mathrm{e}^{\left(\lambda_{i}-\lambda_{j}\right) \tau}=1, \quad \forall i, j \in\{1, \ldots, n\}, i \neq j
$$

is satisfied, then there exist gains $K$ such that $\Delta(K)=0$, and, consequently, Problem 1 with $T=3 \tau$ is solvable;

3. In the single input case, Problem 1 with $T=$ $3 \tau$ is solvable if and only if (9) is satisfied.

Proof. Let $U=\left[u_{1}, \cdots, u_{n}\right]$, with $u_{i}$ the left eigenvector of $A$ corresponding to eigenvalue $\lambda_{i}, i=$ $1, \ldots, n$, and let $\Lambda=\operatorname{diag}\left(\lambda_{1}, \ldots, \lambda_{n}\right)$. Then we have

$$
U^{\mathrm{H}} A=\Lambda U^{\mathrm{H}},
$$

(here $U^{\mathrm{H}}$ denotes the conjugate transpose of $U$ ) which implies

$$
\begin{aligned}
A & =\left(U^{\mathrm{H}}\right)^{-1} \Lambda U^{\mathrm{H}}, \\
\mathrm{e}^{A t} & =\left(U^{\mathrm{H}}\right)^{-1} \mathrm{e}^{\Lambda t} U^{\mathrm{H}} \\
& =\left(U^{\mathrm{H}}\right)^{-1}\left[\begin{array}{ccc}
\mathrm{e}^{\lambda_{1} t} & & \\
& \ddots & \\
& & \mathrm{e}^{\lambda_{n} t}
\end{array}\right] U^{\mathrm{H}} .
\end{aligned}
$$

As a consequence we have

$$
U^{\mathrm{H}} \Delta(K)\left(U^{\mathrm{H}}\right)^{-1}=\mathrm{e}^{\Lambda \tau}\left[\mathrm{e}^{\Lambda \tau}-\int_{0}^{\tau} \mathrm{e}^{-\Lambda s} \tilde{B} \tilde{K} \mathrm{e}^{\Lambda s} \mathrm{~d} s\right],
$$

where

$$
\begin{aligned}
& \tilde{B}=U^{\mathrm{H}} B:=\left[\begin{array}{c}
b_{1}^{\mathrm{H}} \\
\vdots \\
b_{n}^{\mathrm{H}}
\end{array}\right], \\
& \tilde{K}=K\left(U^{\mathrm{H}}\right)^{-1}:=\left[\begin{array}{lll}
\tilde{K}_{1} & \cdots & \tilde{K}_{n}
\end{array}\right] .
\end{aligned}
$$

We note that the controllability of $(A, B)$ is equivalent to the condition

$$
b_{i} \neq 0, \quad i=1, \ldots, n .
$$

To see this, if $b_{j}=0$ for some $j$, then we have for $L \in \mathbb{R}^{m \times n}$ that $u_{j}^{\mathrm{H}}\left(\lambda_{j} I_{n}-A-B L\right)=u_{j}^{\mathrm{H}}\left(\lambda_{j} I_{n}-A\right)=0$, implying that eigenvalue $\lambda_{j}$ cannot be changed by feedback and is thus uncontrollable. Conversely, if (11) holds then the controllability matrix $C(A, B)$ satisfies

$$
U^{\mathrm{H}} C(A, B)=\left[\begin{array}{ccc}
b_{1}^{\mathrm{H}} & & \\
& \ddots & \\
& & b_{n}^{\mathrm{H}}
\end{array}\right]\left(V_{n} \otimes I_{m}\right),
$$

where

$$
V_{n}=\left[\begin{array}{ccccc}
1 & \lambda_{1} & \lambda_{1}^{2} & \cdots & \lambda_{1}^{n-1} \\
\vdots & & & & \vdots \\
1 & \lambda_{n} & \lambda_{n}^{2} & \cdots & \lambda_{n}^{n-1}
\end{array}\right]
$$

is an (invertible) Vandermonde matrix, inducing $\operatorname{rank} C(A, B)=n$.

The integrand in (10) can be written as

$$
\left[\begin{array}{ccc}
b_{1}^{\mathrm{H}} & & \\
& \ddots & \\
& & b_{n}^{\mathrm{H}}
\end{array}\right]\left(M \otimes I_{m}\right)\left[\begin{array}{ccc}
\tilde{K}_{1} & & \\
& \ddots & \\
& & \tilde{K}_{n}
\end{array}\right] \text {, }
$$

where $M=\left[M_{i j}\right] \in \mathbb{C}^{n \times n}$ is such that

$$
M_{i j}=\left\{\begin{array}{cc}
\frac{1-\mathrm{e}^{-\left(\lambda_{i}-\lambda_{j}\right) \tau}}{\lambda_{i}-\lambda_{j}}, & i \neq j, \\
\tau, & i=j .
\end{array}\right.
$$

Problem 1 is solvable with $T=3 \tau$ if and only if there exist gains $\tilde{K}$ such that monodromy matrix (10) is zero, which can be rephrased using (12) as

$$
b_{i}^{\mathrm{H}} \tilde{K}_{i}=\frac{\mathrm{e}^{\lambda_{i} \tau}}{\tau}, i=1, \ldots, n
$$


and

$$
\left(\mathrm{e}^{\left(\lambda_{i}-\lambda_{j}\right) \tau}-1\right) b_{i}^{\mathrm{H}} \tilde{K}_{j}=0, \quad \forall i, j \in\{1, \ldots, n\}, i \neq j .
$$

We now address the assertions of the proposition. First, if condition (8) holds, then (13)-(14) can be formulated as

$$
\tilde{B} \tilde{K}=\frac{1}{\tau} \operatorname{diag}\left(\mathrm{e}^{\lambda_{1} \tau}, \ldots, \mathrm{e}^{\lambda_{n} \tau}\right),
$$

which cannot be solved because the left-hand side has maximum rank $m<n$, while the right-hand side has rank $n$.

Second, if condition (9) holds, then (14) is trivially satisfied, while in what follows we construct gains satisfying (13). Given that (11) holds, we can assume without losing generality that the left eigenvectors are scaled such that $\left\|b_{i}\right\|_{2}=1, i=$ $1, \ldots n$. Then a solution of (13) is given by $\tilde{K}_{i}=$ $b_{i} \frac{\mathrm{e}^{\lambda \tau_{i}}}{\tau}, i=1, \ldots, n$, which can be expressed in the original coordinates as

$K=\frac{1}{\tau} B^{\mathrm{T}} U \mathrm{e}^{\Lambda \tau} U^{\mathrm{H}}=\frac{1}{\tau} B^{\mathrm{T}} U U^{\mathrm{H}} \mathrm{e}^{A \tau}=\frac{1}{\tau} B^{\mathrm{T}} \mathfrak{R}\left(U U^{\mathrm{H}}\right) \mathrm{e}^{A \tau}$.

Finally, we consider the reverse implication for the single input case $(m=1)$. Assume that equations (13)-(14) are solvable. From the first equation, nonzero scalars $K_{i}$ can be determined. The second equation then implies (9).

On the one hand, the condition (9) is very restrictive, since it implies that all eigenvalues of matrix $A$ must lie on a vertical line in the complex plane, where the distances between eigenvalues are multiples of $\frac{2 \pi}{\tau}$ (yet Proposition 2 will be instrumental in the design approach presented in Section 4.2). On the other hand, imposing a zero monodromy matrix, as control law (6) does, is more than necessary for fixed-time stability, see Proposition 1. Hence, the reported work is driven by the question whether relaxing the requirement on the feedback to render the monodromy matrix only nilpotent is sufficient to result in a feasible solution of the form (2).

Additional motivation lies in the classical pole placement problem using state feedback. Under the controllable assumption of a pair $\left(A_{\mathrm{d}}, B_{\mathrm{d}}\right)$ all eigenvalues of $A_{\mathrm{d}}+B_{\mathrm{d}} K$ can be assigned to zero. The resulting closed-loop matrix is then nilpotent but, except for degenerate cases, not identically zero.

\section{Fixed-time stabilization of planar systems}

The following proposition states that fixed-time stability can always be reached for planar systems.

Proposition 3. Assume $A \in \mathbb{R}^{2 \times 2}$ and $B \in \mathbb{R}^{2 \times 1}$. Then there always exists a controller of the form
(3) such that monodromy matrix $\Delta$ is nilpotent. Consequently, Problem 1 is solvable with convergence time $T=5 \tau$.

Proof. We distinguish between two cases.

Case 1 Matrix A is diagonalizable

Let $\mathcal{N}$ correspond to the right-hand side of (10). Setting $L_{i}=\bar{b}_{i} \tilde{K}_{i} \tau \mathrm{e}^{\lambda_{i} \tau}, i=1,2$, and following the notation as in the proof of Proposition 2, the elements of $\mathcal{N}$ satisfy

$$
\begin{aligned}
& \mathcal{N}_{11}=\mathrm{e}^{2 \lambda_{1} \tau}-L_{1}, \\
& \mathcal{N}_{22}=\mathrm{e}^{2 \lambda_{2} \tau}-L_{2}, \\
& \mathcal{N}_{12} \mathcal{N}_{21}=\frac{1}{\tau^{2}} M_{12} M_{21} L_{1} L_{2} .
\end{aligned}
$$

Matrix $\mathcal{N}$ is nilpotent if and only if $\mathcal{N}_{22}=-\mathcal{N}_{11}$ and $\mathcal{N}_{11} \mathcal{N}_{22}-\mathcal{N}_{12} \mathcal{N}_{21}=0$, which translates into the conditions

$$
\begin{gathered}
L_{1}+L_{2}=\mathrm{e}^{2 \lambda_{1} \tau}+\mathrm{e}^{2 \lambda_{2} \tau}, \\
\alpha L_{1} L_{2}+L_{1} \mathrm{e}^{2 \lambda_{2} \tau}+L_{2} \mathrm{e}^{2 \lambda_{1} \tau}-\mathrm{e}^{2 \lambda_{1} \tau} \mathrm{e}^{2 \lambda_{2} \tau}=0,
\end{gathered}
$$

with

$$
\alpha:=\frac{1}{\tau^{2}} M_{12} M_{21}-1 .
$$

Substituting $L_{2}=\mathrm{e}^{2 \lambda_{1} \tau}+\mathrm{e}^{2 \lambda_{2} \tau}-L_{1}$ into the second equation of (15), we obtain the quadratic equation

$$
\begin{gathered}
F\left(L_{1}\right):=-\alpha L_{1}^{2}+ \\
\left(\alpha\left(\mathrm{e}^{2 \lambda_{1} \tau}+\mathrm{e}^{2 \lambda_{2} \tau}\right)+\mathrm{e}^{2 \lambda_{2} \tau}-\mathrm{e}^{2 \lambda_{1} \tau}\right) L_{1}+\mathrm{e}^{4 \lambda_{1} \tau}=0 .
\end{gathered}
$$

We can express $\alpha=g\left(\left(\lambda_{1}-\lambda_{2}\right) \tau\right)$, with

$$
\begin{aligned}
g(x) & =\frac{\left(1-\mathrm{e}^{-x}\right)\left(1-\mathrm{e}^{x}\right)}{}-1 \\
& =2\left(\frac{x^{2} x^{2}}{4 !}+\frac{x^{4}}{6 !}+\frac{x^{6}}{8 !}+\cdots\right) .
\end{aligned}
$$

In what follows we make a further subdivision, based on the spectrum of $A$.

Case la Real eigenvalues

All coefficients in equation $F\left(L_{1}\right)=0$ are real valued. In addition, we have $\alpha>0$ and $F(0)>$ 0 , from which we conclude that $F$ has real valued zeros, hence, equations (15) are solvable.

Case $1 b$ A complex conjugate pair of eigenvalues

We can assume, without losing generality, that $A$ takes the form

$$
A=\left[\begin{array}{rr}
\sigma & \omega \\
-\omega & \sigma
\end{array}\right],
$$

with eigenvalues $\lambda_{1,2}=\sigma \pm \mathrm{i} \omega$. Let $x=\mathfrak{R}\left(\mathrm{e}^{2 \lambda_{1} \tau}\right)$ and $y=\mathfrak{J}\left(\mathrm{e}^{2 \lambda_{1} \tau}\right)$. Then we have both $\mathrm{e}^{2 \lambda_{1} \tau}=x+\mathrm{i} y$ and $\mathrm{e}^{2 \lambda_{2} \tau}=x-\mathrm{i} y$. The first equation of (15) reads as

$$
L_{1}+L_{2}=2 x \text {. }
$$


Equation (16) can be written as

$$
-\alpha L_{1}^{2}+(2 \alpha x-\mathrm{i} 2 y) L_{1}+(x-\mathrm{i} y)^{2}=0,
$$

with solutions

$$
L_{1}^{ \pm}=\frac{-2 \alpha x+\mathrm{i} 2 y \pm 2 \sqrt{\alpha(1+\alpha) x^{2}-(1+\alpha) y^{2}}}{-2 \alpha} .
$$

For $v \in \mathbb{R}$, we have

$$
g(\mathrm{i} v)=\left(\operatorname{sinc}\left(\frac{v}{2 \pi}\right)\right)^{2}-1,
$$

which implies

$$
\alpha=g\left(\left(\lambda_{1}-\lambda_{2}\right) \tau\right) \in[-1,0) .
$$

As a consequence, the argument of the square root in (19) is non-positive and $\mathfrak{R}\left(L_{1}^{ \pm}\right)=x$. Combining this result with (18) we find that the solution pair $\left(L_{1}^{+}, L_{2}^{+}\right)$consists of complex conjugate numbers (the same holding for the other solution pair).

Recall that expressions (15) are based on a diagonalization of the system. In the coordinate system where the system matrix takes the form (17), we get

$$
\begin{aligned}
K & =\left[\begin{array}{cc}
\tilde{K}_{1} & \tilde{K}_{2}
\end{array}\right] U^{\mathrm{H}} \\
& =\left[\begin{array}{ll}
\frac{L_{1}^{+}}{\overline{b_{1}} \mathrm{\tau}^{\mathrm{e}_{1} \tau}} & \frac{L_{2}^{+}}{\overline{b_{2}} \mathrm{\tau}^{\mathrm{e}_{2} \tau}}
\end{array}\right] U^{\mathrm{H}} .
\end{aligned}
$$

Since we have $L_{1}^{+}=\overline{L_{2}^{+}}, b_{1}=\bar{b}_{2}, \lambda_{1}=\bar{\lambda}_{2}$ and the matrix of left eigenvectors can be chosen as

$$
U=\left[\begin{array}{rr}
1 & 1 \\
\mathrm{i} & -\mathrm{i}
\end{array}\right],
$$

we conclude that $K$ is real valued.

\section{Case 2 Matrix A is not diagonalizable}

In this case matrix $A$ has a double eigenvalue on the real axis, and we consider without losing generality

$$
A=\left[\begin{array}{rr}
\lambda_{0} & 1 \\
0 & \lambda_{0}
\end{array}\right], B=\left[\begin{array}{l}
\beta_{1} \\
\beta_{2}
\end{array}\right] .
$$

We now have

$$
\mathrm{e}^{A t}=\left[\begin{array}{rr}
\mathrm{e}^{\lambda_{0} t} & t \mathrm{e}^{\lambda_{0} t} \\
0 & \mathrm{e}^{\lambda_{0} t}
\end{array}\right] .
$$

Similarly to (15), the conditions for $\Delta$ to be nilpotent can be rephrased as (with $K=\left[K_{1} K_{2}\right]$ )

$$
\left\{\begin{array}{l}
2 \mathrm{e}^{\lambda_{0} \tau}+\left(-\beta_{1} \tau-\beta_{2} \tau^{2}\right) K_{1}-\beta_{2} \tau K_{2}=0 \\
\mathrm{e}^{2 \lambda_{0} \tau}+\frac{\beta_{2}^{2} \tau^{4}}{12} K_{1}^{2}+\left(\beta_{2} \tau^{2} \mathrm{e}^{\lambda_{0} \tau}-\right. \\
\left.\beta_{1} \tau \mathrm{e}^{\lambda_{0} \tau}\right) K_{1}-\beta_{2} \tau \mathrm{e}^{\lambda_{0} \tau} K_{2}=0 .
\end{array}\right.
$$

Eliminating $K_{2}$ results in the quadratic equation

$$
\frac{\beta_{2}^{2} \tau^{4}}{12} K_{1}^{2}+2 \beta_{2} \tau^{2} \mathrm{e}^{\lambda_{0} \tau} K_{1}-\mathrm{e}^{2 \lambda_{0} \tau}=0 .
$$

From the controllability condition we have $\beta_{2} \neq 0$. The product of the solutions is given by

$$
-\frac{12 \mathrm{e}^{2 \lambda_{0} \tau}}{\beta_{2}^{2} \tau^{4}}<0
$$

hence, they must lie on the real axis.

Remark 1. As (16) was shown to have two real solutions and (21) two real zeros, there always exist two isolated solutions for $K$, that solve Problem 1 .

Remark 2. If A has an eigenvalue in the open right half plane, then system

$$
\dot{x}(t)=A x(t)-B K x(t-\tau)
$$

is always unstable for sufficiently large $\tau$ (see, e.g. [13]), while in Proposition 3 no conditions are put on the delay. Hence, for large delay the (fixedtime) stabilizing control law (2) leads to an alternation of the closed-loop dynamics between unstable system (22) and the unstable open loop system, while the stability is induced by the time-dependent switching.

If $A$ has a complex conjugate pair of eigenvalues,

$$
\lambda_{1,2}=\sigma \pm \mathrm{j} \omega, \omega \neq 0,
$$

it is also possible to introduce an additional delay

$$
\delta \tau=\frac{\pi}{\omega}\left(\left\lceil\frac{\omega \tau}{\pi}\right\rceil-\frac{\omega \tau}{\pi}\right)
$$

in the feedback loop such that the pair $(\omega, \tau+\delta \tau)$ satisfies

$$
\mathrm{e}^{\left(\lambda_{1}-\lambda_{2}\right)(\tau+\delta \tau)}=1,
$$

which is key in invoking Proposition 2. Consider the control law

$$
u(t)=-g_{\tau+\delta \tau}(t) K x(t-\delta \tau),
$$

which is well defined for $t \geq \delta \tau$ while for $t \in$ $[0, \delta \tau)$, we have $g_{\tau+\delta \tau}(t)=0$ and we can simply set $u(t)=0$. We can now state the following result.

Proposition 4. Assume that the eigenvalues of $A$ are given by (23) and let $\delta \tau$ be defined by (24). Then there always exists a controller of the form (26) such that monodromy matrix $\Delta$ is zero. Consequently, fixed-time stability with convergence time $T=3 \tau+2 \delta \tau$ is achieved.

Proof. For $t \geq \tau$ the closed-loop system is described by

$$
\dot{x}(t)=A x(t)-g_{\tau+\delta \tau}(t-\tau) K x(t-(\tau+\delta \tau)) .
$$

The monodromy matrix mapping $x(\tau)$ to $x(\tau+2(\tau+$ $\delta \tau)$ ) can be made zero, following from condition (25) and Proposition 2. This implies $x(t)=0$ for $t \geq 3 \tau+2 \delta \tau$. 


\section{General case}

In terms of the characteristic function of the monodromy matrix,

$$
\begin{aligned}
& \left|s I_{n}-\Delta(K)\right|= \\
& s^{n}+\alpha_{n-1}(K) s^{n-1}+\cdots+\alpha_{1}(K) s+\alpha_{0}(K),
\end{aligned}
$$

the conditions on gain $K$ to render $\Delta$ nilpotent are

$$
\alpha_{0}(K)=\cdots=\alpha_{n-1}(K)=0 \text {. }
$$

Constructing and solving this system of equations analytically, on which the proof of Proposition 3 relies, is quickly infeasible if dimension $n$ is increased.

1. First, this approach relies on symbolically computing the characteristic equation. The latter results in a system of polynomial equations (notice that $\alpha_{i}$ is a polynomial of joint degree $n-i$ in the elements of $K$, for $i=$ $0, \ldots, n-1)$. Methods for constructing and solving such a system of equations symbolically (e.g. using Groebner bases or resultant theory) scale badly with respect to the degree and number of variables.

2. Second, the solutions of interest of the polynomial equations are restricted to be real valued.

3. Finally, the analysis for planar systems revealed that distinct isolated solutions may exist (see Remark 1), indicating that convex reformulations do not exist.

Therefore, we proceed in two complementary directions. First, in Section 4.1 we present an algorithm to numerically compute gain $K$ rendering $\Delta$ nilpotent. This algorithm is generally applicable and effective in practice, yet the existence of and convergence to a feasible solution is not supported by mathematical proofs. Second, in Section 4.2 we demonstrate that fixed-time stability can always be guaranteed by a modified control law, relying on a combination of Artstein's transformation, an additional feedback transformation and Proposition 2.

\subsection{A smooth optimization based algorithm}

In order to avoid computing the characteristic equation explicitly, a standard approach from an eigenvalue optimization point of view would be to solve the optimization problem

$$
\min _{K} \rho(\Delta(K)),
$$

with $\rho(\cdot)$ the spectral radius, since $\Delta$ is nilpotent if and only if $\rho(\Delta)=0$. However, this problem is a non-smooth optimization problem and the objective function is typically non-Lipschitz in the global minimizers (note that in the generic case the index of nilpotency of $\Delta(K)$ is larger than one).

The following simple fact, which is a consequence of the well-known Leverrier-Faddeev algorithm, allows to avoid solving a non-smooth problem. A proof is provided for completeness.

Lemma 1. Let the characteristic function of $\Delta$ be given by (27). Then

$$
\left[\begin{array}{c}
\operatorname{tr}(\Delta) \\
\operatorname{tr}\left(\Delta^{2}\right) \\
\operatorname{tr}\left(\Delta^{3}\right) \\
\vdots \\
\operatorname{tr}\left(\Delta^{n}\right)
\end{array}\right]=-S_{n}^{-1}\left[\begin{array}{c}
\alpha_{n-1} \\
2 \alpha_{n-2} \\
3 \alpha_{n-3} \\
\vdots \\
n \alpha_{0}
\end{array}\right]
$$

where

$$
S_{n}=\left[\begin{array}{ccccc}
1 & & & & \\
\alpha_{n-1} & 1 & & & \\
\alpha_{n-2} & \alpha_{n-1} & 1 & & \\
\vdots & \vdots & \ddots & \ddots & \\
\alpha_{1} & \alpha_{2} & \cdots & \alpha_{n-1} & 1
\end{array}\right] .
$$

Thus $\Delta$ is nilpotent if and only if $\operatorname{tr}\left(\Delta^{k}\right)=0$ for $k=1,2, \ldots, n$.

Proof. By the well-known Leverrier-Faddeev algorithm, the coefficients of the characteristic function can be computed recursively as

$$
\begin{aligned}
& R_{n-k}=R_{n-k+1} \Delta+\alpha_{n-k+1} I_{n}, \\
& \alpha_{n-k}=-\frac{\operatorname{tr}\left(R_{n-k} \Delta\right)}{k}, k=1,2, \ldots, n,
\end{aligned}
$$

with $R_{n}=0$ and $\alpha_{n}=1$. It follows that, by repeatedly using the above relations,

$$
\begin{aligned}
\alpha_{n-1} & =-\frac{\operatorname{tr}(\Delta)}{1}, \\
\alpha_{n-2} & =-\frac{\operatorname{tr}\left(\Delta^{2}\right)}{2}-\alpha_{n-1} \frac{\operatorname{tr}(\Delta)}{2}, \\
\alpha_{n-3} & =-\frac{\operatorname{tr}\left(\Delta^{3}\right)}{3}-\alpha_{n-1} \frac{\operatorname{tr}\left(\Delta^{2}\right)}{3}-\alpha_{n-2} \frac{\operatorname{tr}(\Delta)}{3}, \\
\vdots & \\
\alpha_{0} & =-\frac{\operatorname{tr}\left(\Delta^{n}\right)}{n}-\alpha_{n-1} \frac{\operatorname{tr}\left(\Delta^{n-1}\right)}{n}-\cdots-\alpha_{1} \frac{\operatorname{tr}(\Delta)}{n},
\end{aligned}
$$

which can be written as (30).

Our algorithm for computing $K$ such that $\Delta(K)$ is nilpotent is based on solving the system of $n$ nonlinear equations $F(K)=0$, spelled out as

$$
F(K)=\left[\begin{array}{c}
\operatorname{tr}(\Delta(K)) \\
\operatorname{tr}\left(\Delta^{2}(K)\right) \\
\vdots \\
\operatorname{tr}\left(\Delta^{n}(K)\right)
\end{array}\right]=0 .
$$


Obviously, function $\mathbb{R}^{m \times n} \ni K \mapsto F(K)$ is real analytic. To evaluate $\Delta(K)$, with $K=\left[K_{i j}\right]$, we get from (7) that

$$
\Delta(K):=\mathrm{e}^{2 A \tau}-\sum_{i=1}^{m} \sum_{j=1}^{n} K_{i j} \int_{0}^{\tau} \mathrm{e}^{A(\tau-s)} B E_{i j} \mathrm{e}^{A s} \mathrm{~d} s,
$$

where $E_{i j}$ is an $m \times n$ matrix whose $(i, j)$-th element is 1 and the others are zero. The Jacobian of $F$ (after vectorizing $K$ ), can be computed using the expression

$$
\begin{aligned}
\frac{\partial \operatorname{tr}\left(\Delta^{k}\right)}{\partial K_{i j}} & =\operatorname{tr}\left(\frac{\partial \Delta^{k}}{\partial K_{i j}}\right) \\
& =\operatorname{tr}\left(\sum_{s=0}^{k-1} \Delta^{s} \frac{\partial \Delta}{\partial K_{i j}} \Delta^{k-1-s}\right) \\
& =\operatorname{tr}\left(\sum_{s=0}^{k-1} \frac{\partial \Delta}{\partial K_{i j}} \Delta^{s} \Delta^{k-1-s}\right) \\
& =\operatorname{tr}\left(\Delta^{k-1} \sum_{s=0}^{k-1} \frac{\partial \Delta}{\partial K_{i j}}\right) \\
& =k \operatorname{tr}\left(\Delta^{k-1} \frac{\partial \Delta}{\partial K_{i j}}\right),
\end{aligned}
$$

and, inferred from (7),

$$
\begin{aligned}
\frac{\partial \Delta(K)}{\partial K_{i j}} & =-\int_{0}^{\tau} \mathrm{e}^{A(\tau-s)} B \frac{\partial K}{\partial K_{i j}} \mathrm{e}^{A s} \mathrm{~d} s \\
& =-\int_{0}^{\tau} \mathrm{e}^{A(\tau-s)} B E_{i j} \mathrm{e}^{A s} \mathrm{~d} s .
\end{aligned}
$$

It follows that both the coefficient matrices of $K_{i j}$ in (32) and matrices $\frac{\partial \Delta}{\partial K_{i j}}$ do not depend on the gain $K$ and need to be numerically evaluated only once.

For the single input case, our code ${ }^{1}$ is based on a trust region dogleg algorithm for solving (31), which employs as search direction a convex combination of the Newton-Raphson step and a step in the direction of steepest descent for objective function

$$
J(K)=\sum_{k=1}^{n}\left(\operatorname{tr}\left(\Delta^{k}(K)\right)\right)^{2}
$$

For the multiple input case, where the equations are over-determined, the Levenberg-Marquardt algorithm is used to minimize the residual norm (34), as implemented in the MATLAB function fsolve.

\subsection{Predictor based feedback}

The fixed time-stabilization of problem (1) can be transformed into the corresponding problem for

\footnotetext{
${ }^{1}$ Available for download using http://twr.cs.kuleuven.be/research/software/delay-control/ stabfinite.m
}

a linear system without input delay by the so-called predictor feedback. To this end, we define $[1,9]$

$$
w(t):=\mathrm{e}^{A \tau} x(t)+\int_{t}^{t+\tau} \mathrm{e}^{A(t+\tau-s)} B \bar{u}(s-\tau) \mathrm{d} s,
$$

where $t \geq 0$ and

$$
\bar{u}(t):=\left\{\begin{array}{l}
u(t), \forall t \geq 0, \\
\bar{v}(t), \forall t \in[-\tau, 0),
\end{array}\right.
$$

in which $\bar{v}(t)$ is an arbitrarily bounded function. The following lemma is well known.

Lemma 2. [1] For $t \geq \tau$ the function $w$ satisfies

$$
\dot{w}(t)=A w(t)+B u(t),
$$

and

$$
w(t)=x(t+\tau),
$$

that is, $w$ takes the role of predictor of state $x$.

The subsequent fixed-time stabilization of (37) can be done in two steps. First we perform a feedback transformation combined with an artificial input delay such that conditions (9) are satisfied. Next, we derive a fixed-time stabilizing control law following the proof of Proposition 2. More precisely, given arbitrary constants

$$
h>0, \quad r>0,
$$

we perform the feedback transformation

$$
u(t)=-K_{0} w(t)+v(t)
$$

transforming (37) to

$$
\dot{w}(t)=\left(A-B K_{0}\right) w(t)+B v(t),
$$

where $K_{0}$ is chosen such that the spectrum of $A-$ $B K_{0}$ satisfies

$$
\begin{gathered}
\Sigma\left(A-B K_{0}\right)= \\
\begin{cases}\left\{-r+k \frac{2 \pi}{h} \mathrm{i}: k=-\frac{n-1}{2}, \ldots, \frac{n-1}{2},\right\}, & \text { if } n \text { is odd, } \\
\left\{-r+\frac{(2 k+1) \pi}{h} \mathrm{i}: k=-\frac{n}{2}, \ldots, \frac{n}{2}-1\right\}, & \text { if } n \text { is even. }\end{cases}
\end{gathered}
$$

Now we employ the additional feedback

$$
v(t)=-g_{h}(t-\tau) K w(t-h),
$$

which is well defined for $t \geq h$, while for $t \in[0, h)$ we can simply set $w(t-h)=0$. From $t \geq \tau$ on, function $w$ satisfies

$$
\dot{w}(t)=\left(A-B K_{0}\right) w(t)-g_{h}(t-\tau) B K w(t-h) .
$$

The combination of delay $h$ in (40) and spectrum (38) assures that condition (9) is satisfied. As a consequence, we conclude from Proposition 2 that 
fixed-time stability can be reached in (additional) time $2 h$. Moreover, from its proof the corresponding gain can be computed as

$$
K=\frac{1}{h} B^{\mathrm{T}} \mathfrak{R}\left(U U^{\mathrm{H}}\right) \mathrm{e}^{\left(A-B K_{0}\right) h} .
$$

Here $U=\left[u_{1} \cdots u_{n}\right]$ collects the left eigenvectors of $\left(A_{0}-B K_{0}\right)$, i.e.

$$
U^{\mathrm{H}}\left(A-B K_{0}\right)=\left[\begin{array}{lll}
\lambda_{1} & & \\
& \ddots & \\
& & \lambda_{n}
\end{array}\right] U^{\mathrm{H}},
$$

with $\Sigma\left(A-B K_{0}\right)=\left\{\lambda_{1}, \ldots, \lambda_{n}\right\}$, where the eigenvectors are scaled such that

$$
\left\|u_{i}^{\mathrm{H}} B\right\|_{2}=1, i=1, \ldots, n .
$$

Finally, by Lemma 2 the property $w(t)=0$ for $t \geq$ $\tau+2 h$ implies $x(t)=0$ for $t \geq 2 \tau+2 h$. This brings us to the following result.

Proposition 5. For $h>0$ and $r>0$, let $K_{0}$ be computed according to (38) and $U$ according to (41)-(42). Then the control law

$$
\begin{aligned}
& u(t)=-K_{0} w(t) \\
& -\frac{1}{h} g_{h}(t-\tau) B^{\mathrm{T}} U U^{\mathrm{H}} \mathrm{e}^{\left(A-B K_{0}\right) h} w(t-h),
\end{aligned}
$$

with $w$ defined by (35), achieves fixed-time stability of (1) with convergence time $T=2 \tau+2 h$.

\section{Illustrations}

\subsection{Local model for an inverted pendulum}

We consider the dimensionless linearized model for an inverted pendulum on a position-controlled cart, considered in Section 4.1 of [4], for which

$$
A=\left[\begin{array}{cc}
0 & 1 \\
2.5 & 0
\end{array}\right], B=\left[\begin{array}{l}
0 \\
1
\end{array}\right], \tau=1
$$

Proposition 3 states that fixed-time stability with convergence time $T=5$ can always be achieved with controller (2), and from its constructive proof the two isolated solutions for the gain can be computed as

$$
K=\left[\begin{array}{ll}
7.6992 & 4.8690
\end{array}\right] \text {, }
$$

and

$$
K=\left[\begin{array}{ll}
-343.80 & 209.12
\end{array}\right] \text {. }
$$

In Figures 1-2 we show contour lines of the spectral radius of the monodromy matrix $\rho(\Delta(K))$ as a function of $K$, around (45) and (46), from which we make two observations. First, the shape of the contour lines illustrates the non-smoothness of the

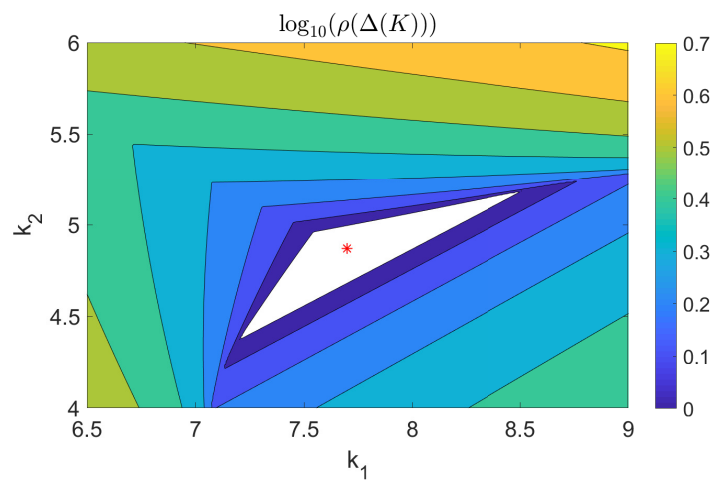

Figure 1: Contour lines of the spectral radius of the monodromy matrix of (1) and (2) as a function of $K=\left[k_{1}, k_{2}\right]$, corresponding to (44). The stability region is colored white. Gain (45) is indicated with an asterisk.

spectral radius, which motivates to solve system (31) rather than optimization problem (29) in a numerical approach. Second, while the stability region around (45) is considerable, the one around (46) is tiny, resulting in a lack of robustness against parametric uncertainty.

For gain (45) we show in Figure 3 the solution of the closed-loop system for initial condition $x_{0}=[1,1]^{\mathrm{T}}$ and $u_{0}=0$. As expected from Proposition 3 , the solution settles down to zero in time $5 \tau=5$.

\subsection{A chain of integrators}

We consider a chain of $n$ integrators with input delay, such that

$$
A=\left[\begin{array}{ccccc}
0 & 1 & 0 & \cdots & 0 \\
\vdots & & 1 & & \vdots \\
\vdots & & & \ddots & \\
& & & & 1 \\
0 & & \cdots & & 0
\end{array}\right], B=\left[\begin{array}{c}
0 \\
0 \\
\vdots \\
0 \\
1
\end{array}\right], \tau=1 .
$$

For $n \in\{2, \ldots, 10\}$, we executed the numerical algorithm described in Section 4.1, with the zero vector as initial condition for the gain $K$. The results are visualized in Table 1 . The second and third column display the residual norm of equations (31), evaluated in the computed solution $K_{*}$, and the spectral norm of $\Delta^{n}\left(K_{*}\right)$. In order to get strong numerical indications that the computed gains are accurate approximations of gains that effectively render $\Delta$ nilpotent, we performed two complementary checks.

The first one is based on assessing the numerical condition of the problem. For this, we show in the fourth column of Table 1 the quantity $\frac{\left\|J_{F}^{-1}\left(K_{*}\right)\right\|_{2}}{\left\|K_{*}\right\|_{2}}$ with $J_{F}$ the Jacobian of $F$ w.r.t. the elements of $K$. If the linearization employed in Newton's method 


\begin{tabular}{|l|l|l|l|l|l|}
\hline $\mathrm{n}$ & $\left\|F\left(K_{*}\right)\right\|_{2}$ & $\left\|\Delta^{n}\left(K_{*}\right)\right\|_{2}$ & $\frac{\left\|J_{F}^{-1}\left(K_{*}\right)\right\|_{2}}{\left\|K_{*}\right\|_{2}}$ & $\rho\left(\Delta\left(K_{*}\right)\right)$ & $\left(\epsilon_{\text {mach }}\right)^{\frac{1}{n}}$ \\
\hline \hline 2 & $6.0019 \mathrm{e}-15$ & $3.1132 \mathrm{e}-15$ & $5.4122 \mathrm{e}-01$ & $5.4781 \mathrm{e}-08$ & $1.0537 \mathrm{e}-08$ \\
3 & $3.0996 \mathrm{e}-15$ & $3.2725 \mathrm{e}-15$ & $3.8899 \mathrm{e}-01$ & $7.7786 \mathrm{e}-06$ & $4.8062 \mathrm{e}-06$ \\
4 & $4.6365 \mathrm{e}-15$ & $5.8125 \mathrm{e}-15$ & $3.1238 \mathrm{e}-01$ & $1.2091 \mathrm{e}-04$ & $1.0265 \mathrm{e}-04$ \\
5 & $1.0964 \mathrm{e}-10$ & $1.0966 \mathrm{e}-10$ & $2.6634 \mathrm{e}-01$ & $7.1734 \mathrm{e}-03$ & $6.4429 \mathrm{e}-04$ \\
6 & $1.6544 \mathrm{e}-13$ & $9.6921 \mathrm{e}-13$ & $2.3582 \mathrm{e}-01$ & $4.0108 \mathrm{e}-03$ & $2.1923 \mathrm{e}-03$ \\
7 & $2.6366 \mathrm{e}-13$ & $6.4791 \mathrm{e}-13$ & $2.1430 \mathrm{e}-01$ & $7.2869 \mathrm{e}-03$ & $5.2574 \mathrm{e}-03$ \\
8 & $3.0999 \mathrm{e}-11$ & $3.1170 \mathrm{e}-10$ & $1.9847 \mathrm{e}-01$ & $3.3499 \mathrm{e}-02$ & $1.0132 \mathrm{e}-02$ \\
9 & $2.9239 \mathrm{e}-12$ & $3.4288 \mathrm{e}-11$ & $1.8649 \mathrm{e}-01$ & $3.9344 \mathrm{e}-02$ & $1.6876 \mathrm{e}-02$ \\
10 & $8.8299 \mathrm{e}-11$ & $3.7071 \mathrm{e}-10$ & $1.7721 \mathrm{e}-01$ & $7.8411 \mathrm{e}-02$ & $2.5383 \mathrm{e}-02$ \\
\hline
\end{tabular}

Table 1: Results of solving equations (31), which describe that $\Delta(K)$ is a nilpotent matrix.
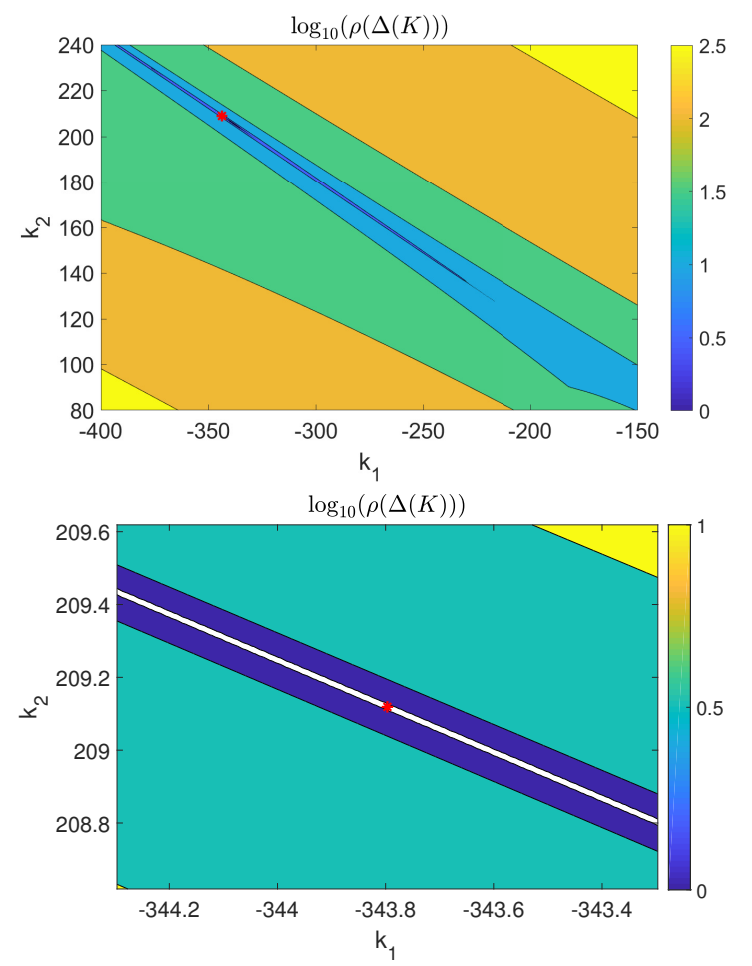

Figure 2: Contour lines of the spectral radius of the monodromy matrix of (1) and (2) as a function of $K=\left[k_{1}, k_{2}\right]$, corresponding to (44). The lower pane corresponds to a zoom of the upper pane around the stability region, which is colored white. Gain (46) is indicated with an asterisk.

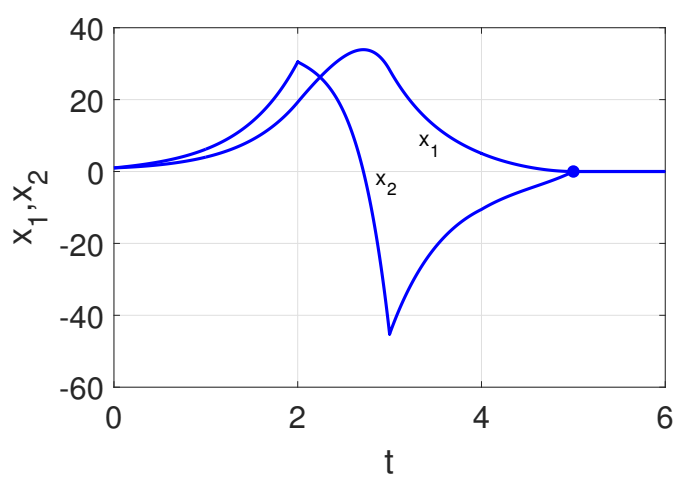

Figure 3: Trajectory of (1) and (2), for model (44) and gain (45).

is accurate, the deviation $\delta K$ of the computed gain from an actual solution to (31) approximately satisfies (after vectorizing $K$ )

$$
\begin{aligned}
& J_{F}\left(K_{*}\right) \delta K=-F\left(K_{*}\right) \\
& \Rightarrow \frac{\|\delta K\|_{2}}{\left\|K_{*}\right\|_{2}} \leq\left(\frac{\left\|J_{F}^{-1}\left(K_{*}\right)\right\|_{2}}{\left\|K_{*}\right\|_{2}}\right)\left\|F\left(K_{*}\right)\right\|_{2} .
\end{aligned}
$$

The fact that the numbers in the fourth column of Table 1 are small, and in particular that they do not increase with respect to dimension $n$, are reassuring for the presence of a nearby solution to Problem 1.

The second check is based on correlating the a-posteriori computed eigenvalues of $\Delta\left(K_{*}\right)$ (its spectral radius is shown in the fifth column of Table 1) with the perturbation theory for eigenvalue problems. For all $n$, the radius $\rho\left(\Delta^{n-1}\left(K_{*}\right)\right)$ is not close to zero, while an index of nilpotency equal $n$ implies that the zero eigenvalue has algebraic multiplicity $n$ and geometric multiplicity one. To such an eigenvalue the following result, adapted from Theorem 2.2 of [12], applies.

Proposition 6. Consider an eigenvalue problem

$$
M(\lambda ; \epsilon) v=0, \lambda \in \mathbb{C}, v \in \mathbb{C}^{n}, v \neq 0,
$$


parameterized by a real parameter $\epsilon$. Assume there exists an open interval $I \subset \mathbb{R}$, containing zero, such that the following properties are satisfied: for all $\epsilon \in I$ the entries of $M$ are entire functions of $\lambda$, for all $\lambda \in \Omega$ the entries of $M$ are smooth functions of $\epsilon$ on $I$ and for all $\epsilon \in I$, the eigenvalue problem is not singular. Let $\lambda_{0}$ be an eigenvalue of (48) for $\epsilon=0$, with algebraic multiplicity equal to $m$ and geometric multiplicity one, with Jordan chain $\left(H_{0}, \ldots, H_{m-1}\right)$. Let $U_{0}$ be the corresponding left eigenvector. Assume that condition

$$
U_{0}^{*} \frac{\partial M}{\partial \epsilon} H_{0} \neq 0
$$

holds. Then around $\epsilon=0$, the eigenvalues in the vicinity of $\lambda_{0}$ can be expanded as the branches of the Puiseux series

$$
\lambda^{(k)}(\epsilon)=\lambda_{0}+\sum_{i=1}^{\infty} \epsilon^{\frac{i}{m}} \lambda_{i}^{(k)},
$$

where

$$
\lambda_{1}^{(k)}=\left(-\frac{U_{0}^{*} \frac{\partial M}{\partial \epsilon} H_{0}}{U_{0}^{*} \Pi}\right)^{\frac{1}{n}} \mathrm{e}^{\mathrm{i} \frac{(k-1) 2 \pi}{n}}
$$

and

$\Pi=\frac{1}{1 !} \frac{\partial M}{\partial \lambda} H_{m-1}+\frac{1}{2 !} \frac{\partial^{2} M}{\partial \lambda^{2}} H_{m-2}+\cdots+\frac{1}{m !} \frac{\partial^{m} M}{\partial \lambda^{m}} H_{0}$,

for $k=1, \ldots, n$.

Expression (50) states that a small perturbation $\epsilon$ induces a bifurcation of the $n$-th order characteristic root into $n$ single eigenvalues, which are approximately equally spread over a circle in the complex plane with radius proportional to $|\epsilon|^{\frac{1}{n}}$. Assume now that for some gain $K$, matrix $\Delta(K)$ would indeed have a zero eigenvalue with geometric multiplicity one and algebraic multiplicity $n$. In practice, numbers are represented in a discrete, floating point representation and operations are done in finite-precision arithmetic. Rounding errors related to the representation of data, and errors introduced by the computation of $\Delta$ and the execution of the eigenvalue solver ${ }^{2}$, have a similar effect on the computed eigenvalues, as the artificial parameter $\epsilon$ in Proposition 6 does $^{3}$, so one would expect to find approximations equally spread on the unit circle. This is precisely how the computed spectrum of $\Delta\left(K_{*}\right)$ looks like, see Figure 4.

\footnotetext{
${ }^{2}$ For a stable algorithm the effects of the induced errors are comparable to errors on the data.

${ }^{3}$ E.g., the effect of computing the eigenvalues of $\tilde{\Delta}$ instead of $\Delta$, due to rounding errors, can be taken into account by defining $M(\lambda ; \epsilon):=-\lambda I+\Delta+\epsilon \frac{\tilde{\Delta}-\Delta}{\|\tilde{\Delta}-\Delta\|_{2}}$.
}
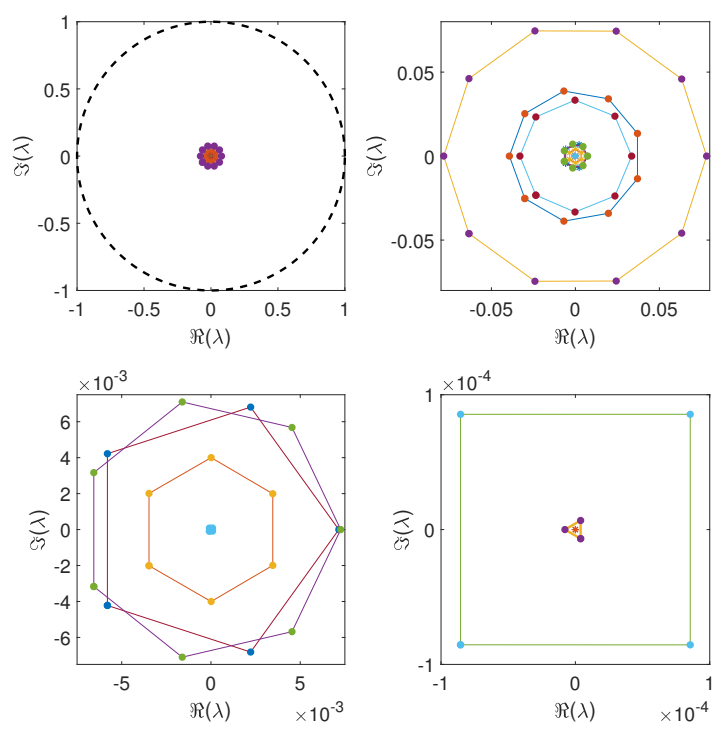

Figure 4: Computed spectrum of $\Delta\left(K_{*}\right)$, corresponding to (47), for different values of $n$ and restricted to different regions of the complex plane (further zoomed in from left to right and from top to bottom).

Furthermore, in the fifth and sixth column of Table 1 we compare the spectral radius $\rho\left(\Delta\left(K_{*}\right)\right)$ with the $n$-th root of the machine precision $\epsilon_{\text {match }}$ (IEEE standard, computations in double precision). As both numbers have the same order of magnitude, we have strong numerical evidence that Problem 1 has been solved, and that a corresponding gain has been computed to full accuracy.

The contrast between the well conditioned problem of computing gains $K$ solving Problem 1 and the high sensitivity of multiple eigenvalues is another motivation to prefer formulation (31) over (29). Important for time-domain interpretations, the norm of $\Delta(K)$ is not very sensitive to perturbations of the gains (see the third column of Table 1)

We now show the result of time-domain simulations for $n=4$. The gain computed from (31) equals

$$
K_{*}=\left[\begin{array}{llll}
0.11787 & 0.60522 & 1.4663 & 2.2114
\end{array}\right],
$$

and for initial conditions $x(0)=[1,1,1,1]^{\mathrm{T}}$ and $u_{0}=0$, the trajectory is shown in Figure 5. The convergence time equals $(2 n+1) \tau=9$.

Finally, we consider the predictor based feedback from Section 4.2, which is theoretically supported by Proposition 5. With the parameter values

$$
r=\frac{1}{16}, h=10,
$$

the feedback transformation is characterized by

$$
K_{0}=\left[\begin{array}{llll}
0.091539 & 0.12435 & 1.0104 & 0.25
\end{array}\right],
$$



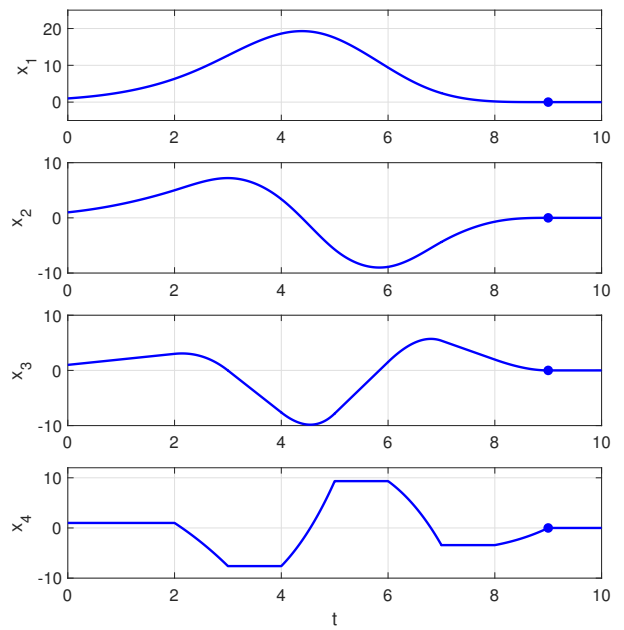

Figure 5: Trajectory of (1) and (2), for model (47) with $n=4$ and gain (52)

and

$$
\Sigma\left(A-B K_{0}\right)=\left\{-\frac{1}{16} \pm \frac{\pi}{10} \mathrm{i},-\frac{1}{16} \pm \frac{3 \pi}{10} \mathrm{i}\right\} .
$$

The control law (39) can then be made explicit as

$$
u(t)=-K_{0} w(t)-K_{D} g_{10}(t-1) w(t-10),
$$

with

$K_{D}=\left[\begin{array}{llll}-0.0066558 & -0.10817 & -0.040145 & -0.21410\end{array}\right]$.

Notice that the second term in the control law, which allows to turn asymptotic stability into fixed-time stability, corresponds to negative feedback. In Figure 6 we depict the solution for initial condition $x(0)=[1,1,1,1]^{\mathrm{T}}$ and $u_{0}=0$, while the predictor (35) is initialized by $\bar{v}(t)=20$ for $t \in[-\tau, 0)$. As expected from the theory, the convergence time equals $T=2 \tau+2 h=22$, while from $t=1 \mathrm{on}, w(t)$ equals $x(t+1)$. The trajectory is seemingly smoother than the one depicted in Figure 5, which can be attributed to the preliminary feedback transformation.

Remark 3. Proposition 5 does not put any assumption on $h$, hence, in theory any convergence time $T>2 \tau$ can be achieved. However, the choice (53) ensures that in an implementation of the predictor (35), the integral term can be approximated by a finite sum (using a quadrature rule) without inducing instability, which requires sufficiently low gains. Note that the potential instability induced by such approximations is a well known problem that traces back to [23].
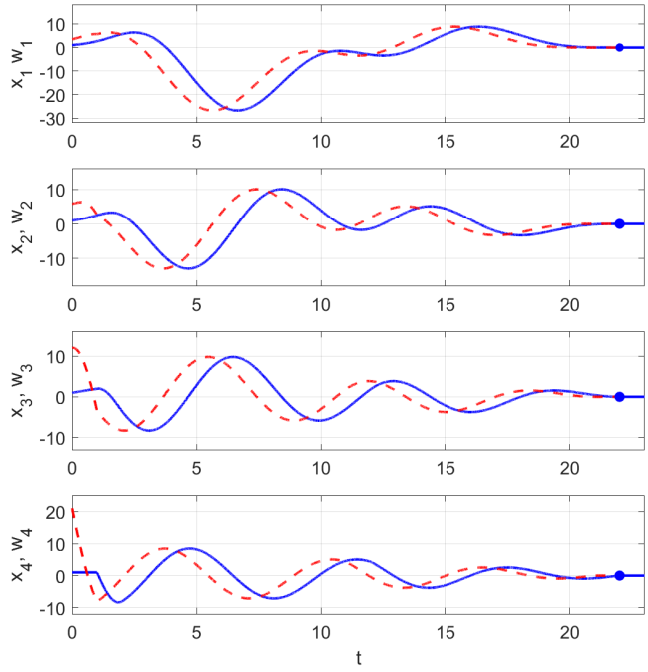

Figure 6: Trajectory of (1), (35) and (54) for model (47) with $n=4$.

\section{Concluding remarks}

We provided both analytical and computational solutions to the fixed-time stabilization problem for input delay systems, which are all grounded in the act-and-wait control principle. The derivations of the analytical results, in particular Propositions 2 and 3, relied on the assumption $m<n$, which is not restrictive. Based on the multivariable control canonical form [11], the design of fixedtime controllers can namely be decoupled into designs for single input systems, while for the scalar case, $\dot{x}(t)=a x(t)+u(t-\tau)$, the choice $K=\mathrm{e}^{a \tau} / \tau$ in (2) results in $\mathcal{M}=0$ and a convergence-time $3 \tau$.

The paper offers several perspectives for further research. A first direction consists of adapting the numerical approach of Section 4.1 towards eigenvalue assignment for the monodromy matrix, and of theoretically investigating its possibilities and limitations. Another direction consists of extending Proposition 3 towards higher-order systems. For example, for a chain of multiple integrators, the thorough numerical investigation reported in Section 5.2 and the fact that stabilizability using the control law (2) for $\tau=1$ implies stabilizability for any delay (relying on a re-scaling of time), bring us to the following conjecture.

Conjecture 1. For any $n \in \mathbb{N}$ and $\tau \geq 0$, the chain of integrators $\frac{\mathrm{d}^{n} x(t)}{\mathrm{d} t^{n}}=u(t-\tau)$ can be stabilized in fixed time $(2 n+1) \tau$ using a control law of the form (2). 


\section{Acknowledgments}

The authors like to thank dr. Simon Telen, for the interesting discussions on algebraic geometry and, in particular, generalizations of Proposition 3. This work was supported by the project C14/17/072 of the KU Leuven Research Council, by the project G0A5317N of the Research Foundation-Flanders (FWO-Vlaanderen), by the Natural Science Foundation of China under the grant number 61773140, and by the Fundamental Research Funds for the Central Universities.

\section{References}

[1] Z. Artstein. Linear systems with delayed control: A reduction. IEEE Transactions on Automatic Control, 27:869-879, 1982.

[2] S.P. Bhat and D.S. Bernstein. Finite-time stability of continuous autonomous systems. SIAM Journal on Control and Optimization, 38(3):751-766, 2000.

[3] S.P. Bhat and D.S. Bernstein. Geometric homogeneity with applications to finite-time stability. Mathematics of Control, Signals, and Systems, 17:101-127, 2005.

[4] F. Borgioli, D. Hajdu, T. Insperger, G. Stépan, and W. Michiels. Pseudospectral method for assessing stability robustness for linear time-periodic delayed dynamical systems. International Journal for Numerical Methods in Engineering, n/a(n/a).

[5] D. Efimov, A. Polyakov, W. Perruquetti, and J. Richard. Weighted homogeneity for time-delay systems: Finitetime and independent of delay stability. IEEE Transactions on Automatic Control, 61(1):210-215, 2016.

[6] Y. Hong. Finite-time stabilization and stabilizability of a class of controllable systems. Systems $\mathcal{E}$ Control Letters, 46(4):231-236, 2002.

[7] T. Insperger. Act-and-wait concept for continuous-time control systems with feedback delay. IEEE Transactions on Control Systems Technology, 14(5):974-977, 2006.

[8] K. Konishi, H. Kokame, and N. Hara. Delayed feedback control based on the act-and-wait concept. Nonlinear Dynamics, 63:513-519, 2011.

[9] M. Krstić. Delay compensation for nonlinear, adaptive and PDE systems. Birkhauser, 2007.

[10] W. H. Kwon and A. Pearson. A modified quadratic cost problem and feedback stabilization of a linear system. IEEE Transactions on Automatic Control, 22(5), 838842, 1977.

[11] D. Luenberger. Canonical forms for linear multivariable systems. IEEE Transactions on Automatic Control, 12(3):290-293, 1967.

[12] W. Michiels, I. Boussaada, and S.-I. Niculescu. An explicit formula for the splitting of multiple eigenvalues for nonlinear eigenvalue problems and connections with the linearization for the delay eigenvalue problem. SIAM Journal on Matrix Analysis and Applications, 38(2):599620, 2017.

[13] W. Michiels, K. Engelborghs, P. Vansevenant, and D. Roose. The continuous pole placement method for delay equations. Automatica, 38(5):747-761, 2002.

[14] A. Polyakov. Nonlinear feedback design for fixed-time stabilization of linear control systems. IEEE Transactions on Automatic Control, 57(8):2106-2110, 2012.

[15] A. Polyakov, D. Efimov, W. Perruquetti, and J.P. Richard. Implicit Lyapunov-Krasovski functionals for stability analysis and control design of time-delay systems. IEEE Transactions on Automatic Control, 60(12):3344-3349, 2015.
[16] V. Pyragas and K. Pyragas. Act-and-wait time-delayed feedback control of nonautonomous systems. Physical Review E, 94(1):012201, 2016.

[17] Y. Shtessel, C. Edwards, L. Fridman, and A. Levant. Sliding Mode Control and Observation. Springer: New York, 2014.

[18] H. Silm, D. Efimov, W. Michiels, R. Ushirobira, and J.-P. Richard. A simple finite-time distributed observer design for linear time-invariant systems. Systems $\mathcal{E}$ Control Letters, 141:104707, 2020.

[19] H. Silm, R. Ushirobira, D. Efimov, J. Richard, and W. Michiels. A note on distributed finite-time observers. IEEE Transactions on Automatic Control, 64(2):759-766, 2019.

[20] Y. Song, Y. Wang, J. Holloway, and M. Krstić. Timevarying feedback for regulation of normal-form nonlinear systems in prescribed finite time. Automatica, 83:243251, 2017.

[21] G. Stépán and T. Insperger. Stability of time-periodic and delayed systems - a route to act-and-wait control. Annual Reviews in Control, 30(2):159-168, 2006.

[22] Z.-Y. Sun, Y. Shao, and C. C. Chen. Fast finite-time stability and its application in adaptive control of high-order nonlinear system. Automatica, 106:339-348, 2019.

[23] V. Van Assche, M. Dambrine, J. F. Lafay, and J. P. Richard. Implementation of a distributed control law for a class of systems with delay. In Proceedings of the 3nd IFAC Workshop on Time Delay Systems, pages 266-271, Santa-Fe, USA, 2001.

[24] J. Wang and R. Kuske. The influence of parametric and external noise in act-and-wait control with delayed feedback. Chaos, 27(11):114319, 2017.

[25] B. Zhou. Finite-time stabilization of linear systems by bounded linear time-varying feedback. Automatica, 113:108760, 2020.

[26] B. Zhou. Finite-time stability analysis and stabilization by bounded linear time-varying feedback. Automatica, to appear.

[27] B. Zhou, W. Michiels, and J. Chen. Prescribed finite-time stabilization of linear delay systems by smooth periodic delayed feedback. Submitted for publication. 